Nota de investigación

\title{
Evaluación de la metodología de sistemas blandos de la cadena productiva del café en Guerrero
}

\author{
Laura Magnolia Santos González ${ }^{1}$ \\ Luis Alberto Olvera Vargas ${ }^{2 \S}$ \\ Víctor Arturo Maldonado Ruelas ${ }^{1}$ \\ ${ }^{1}$ Maestría en Ciencias en Ingeniería-Universidad Politécnica de Aguascalientes. Paseo San Gerardo 207, \\ Fracc. San Gerardo, Aguascalientes, Aguascalientes, México. CP. 20342. Tel. 4494421400. \\ (magnoliasg@hotmail.com; victor.maldonado@upa.edu.mx). ${ }^{2}$ Cátedra CONACYT-Centro de \\ Investigación y Asistencia en Tecnología y Diseño del Estado de Jalisco, AC. Normalistas 800, Colinas de \\ la Normal, Guadalajara, Jalisco, México. CP. 44270. Tel. 3331093057.
}

${ }^{\S}$ Autor para correspondencia: lolvera@ciatej.mx.

\section{Resumen}

Con el objetivo de realizar una evaluación preliminar de la problemática actual que rodea la producción de café en el estado de Guerrero, se organizó información obtenida de entrevistas en 2019 con productores de los municipios de San Luis Acatlán, Chilpancingo de los Bravos, Chilapa de Álvarez y Atoyac de Álvarez, para reconocer por medio de la metodología de sistemas blandos (SSM) de Checkland, procesos y estrategias que puedan fortalecer la cadena productiva del café en el estado. Los resultados muestran que la producción de café en Guerrero ha disminuido a casi la mitad en los últimos 18 años, afectando a más de 20 mil productores de la región. Las causas y consecuencias son megadiversos, entre las que se mencionan desinterés por parte de los organismos gubernamentales, ausencia de políticas públicas, falta de tecnología y programas para el combate a problemas fitosanitarios. Se identifica que estos problemas se intensifican y cambian conforme la escala temporal y espacial se analizada. El mayor interés que surge por parte de los productores de café en Guerrero es transmitir a las nuevas generaciones el respeto y aprovechamiento del cultivo.

Palabras clave: caficultores, problemática, producción, sistema, valor.

Recibido: abril de 2021

Aceptado: mayo de 2021 
La cadena productiva del café en México ha sufrido cambios a consecuencia de la falta de políticas públicas nacionales e internacionales, que han mermado hasta la plantación y producción; sin embargo, se han tratado de efectuar proyectos integrales desde los años 80's para el desarrollo de complejos agroindustriales que brinden alternativas a los problemas del café rural desde una perspectiva más integral (Santoyo et al., 1995).

Akaki y Huacuja (2006) identificaron que los productores han sido el eslabón más débil de la cadena del café, tanto por la falta de políticas públicas como por el desplome y volatilidad de los precios, mientras que las empresas comercializadoras son los agentes hegemónicos de la cadena. Contreras-Medina et al. (2019); Díaz (2015) mencionan que, para tener una mayor equidad en la distribución de los recursos en la cadena productiva del café, se requieren procesos de innovación formulados a partir de redes de participación incluyentes (sobre todo de mujeres y jóvenes) y autogestión de actores, y a pesar de ser procesos complicados, especialmente en las certificaciones y trazabilidad, han logrado alcanzar la consolidación local y regional.

Guerrero es uno de los estados con problemas en el cultivo de café, en los últimos 20 años disminuyó su producción a casi la mitad, pasando de $68000 \mathrm{t}$ y rendimientos de $1.74 \mathrm{t} \mathrm{ha}^{-1}$ en el año 2000 a 38000 t y rendimientos de 0.94 t ha $^{-1}$ en 2019 (SIAP, 2019). Esta reducción en la producción afecta a más de 20000 productores del estado, que dependen del cultivo y que ahora están comenzando a diversificar sus ingresos en las actividades terciarias incluyendo el comercio.

Además, en el estado faltan apoyos y se tiene baja o nula tecnología para la producción del aromático, así como la poca articulación en áreas productoras de café (Contreras-Medina et al., 2019). Bajo este contexto, este estudio evalúa la situación actual de la cadena productiva del café en el estado de Guerrero, México, a través de la metodología de sistemas blandos Soft Systems Methodology (SSM), por sus siglas en inglés (Checkland, 1999) con el fin de reconocer los procesos y estrategias que fortalezcan la cadena de producción del café.

\section{Metodología de sistemas blandos}

La SSM es útil para dar cuenta de los prejuicios culturales inherentes del observador, en un enfoque culturalmente sensible que puede proporcionar técnicas inclusivas (Wheeler et al., 2000). Checkland (1999); Checkland y Poulter (2006) describen la SSM como una operación del círculo infinito de experiencias para una acción, ya que se presenta como una mejor alternativa para tratar problemas mal definidos, con incertidumbres y abstracciones. Se puede aplicar varias veces a la misma situación hasta que se aclare el problema y esta repetición cíclica impulsa el cambio y la adaptación (Cezarino et al., 2016).

La aplicación de la SSM para la evaluación de la cadena productiva surge como una respuesta a las restricciones del pensamiento sistémico duro en problemas de los sistemas sociales, como lo han sido los programas de apoyo social, que en principio tienen un fuerte contexto político y no contribuyen a resolver el problema de origen (Díaz, 2015).

La investigación por medio de SSM incorpora diferentes modelos para intervenir en el mundo real, permite elegir nuevas soluciones desde diferentes puntos de vista, eligiendo consensuadamente una cuando se resuelve de mejor forma los conflictos de la situación problema, esta repetición cíclica impulsa el cambio y la adaptación (Cezarino et al., 2016). La (Figura 1) muestra la descripción de las etapas de la evaluación de SSM. 


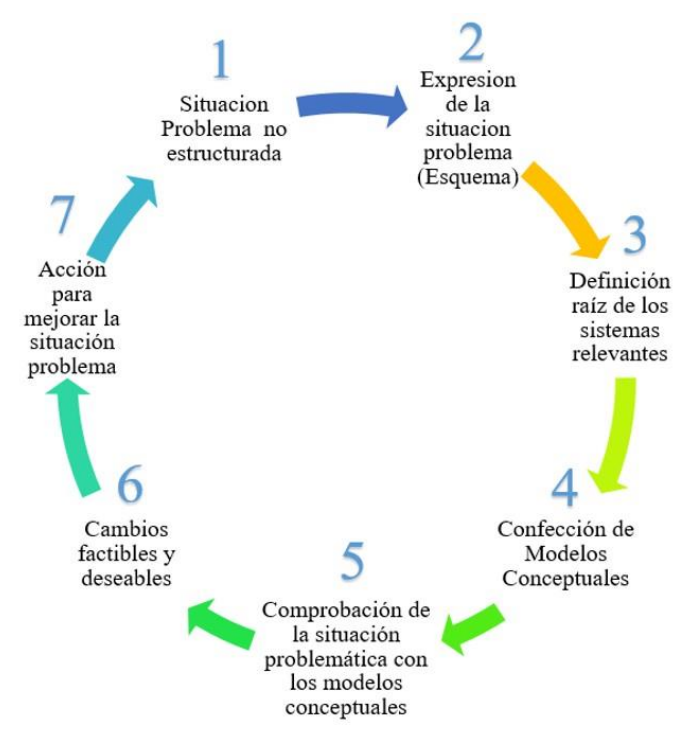

Figura 1. Etapas de la metodología de sistemas blandos (SSM) (Checkland y Poulter, 2006).

En el proceso 1 'Situación problema no estructurada' se identificó que el principal problema del café en el Estado de Guerrero es la caída drástica de producción en 2019, identificando como causas trascendentales la variabilidad climática, la volatilidad de precios internacionales, control de plagas y enfermedades, incremento de violencia, migración y marginación; estas causas reconocen que han afectado a la cadena de producción, desde los procesos de insumos, producción, transformación, comercialización, hasta llegar al consumidor final.

No solo impacta a los actores que realizan dichas actividades, sino que también existe un impacto a nivel nacional por las divisas generadas por la venta del café al exterior. Para mostrar la expresión de la situación problema se realizó un mapa conceptual que se observa en la Figura 2.

La definición de los sistemas relevantes se construyó de las diferentes situaciones problema, comenzando a explorar a los individuos que se relacionan con las actividades del sistema. La falta de tecnología, carente en gran parte del estado, además de la inversión nula por parte del gobierno o apoyos otorgados para ese fin, el proceso realizado al café es natural o capulín, por lo que se vende a un precio muy bajo 'al principio el kilo puede estar cerca o un poco más de $\$ 50.00$ pesos, pero baja a \$20.00 o \$18.00 pesos’ (Flores-López et al., 2018).

Este proceso ha generado que los productores cambien de actividad productiva o sustituya sus cafetales por cultivos más rentables, en otros casos activa la migración de los más jóvenes. Plantaciones viejas, debido a que las plantas de café tienen aproximadamente 50 años o más, sólo se obtiene una cosecha por año, se dificulta las actividades de podar y limpiar los arbustos de cafeto, además, en el estado las plantaciones de café se encuentran rodeadas de otras plantaciones como la guanábana, plátanos y aguacates, plagas y enfermedades, el cafeto es una de las plantas que problemas fitosanitarios puede tener, la roya y la broca de café son las más activas en la zona, además mencionan que la variabilidad climática ha aumentado los problemas de plagas (FloresLópez et al., 2018; Higuera y Rivera, 2018). 


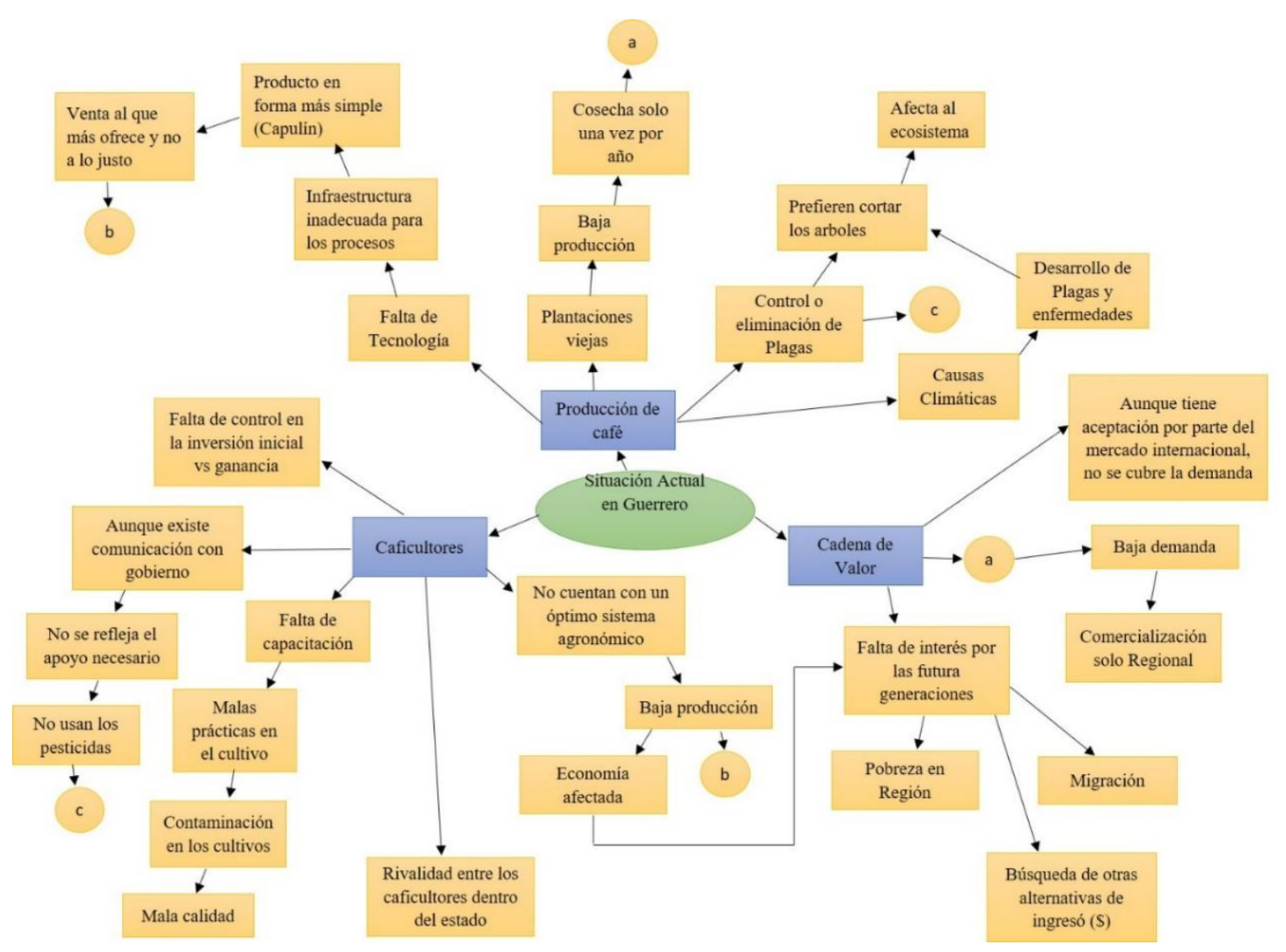

Figura 2. Mapa conceptual de la situación del problema.

Los caficultores confesaron que no suelen hacer constantemente actividades de poda, fertilización, limpieza o deshierbe de las plantaciones de café e incluso utilizan poco los pesticidas que les proporciona el Gobierno del Estado, debido a que no existe capacitación sobre su uso. Pocos o nulos apoyos al cultivo del café y los existentes mal dirigidos o con incumplimiento en sus cláusulas, ejemplo de ello es la iniciativa de Nestlé con su 'código común para la comunidad cafetalera', que los caficultores se expresan que se han 'aprovechado de las necesidades regionales' (Flores-López et al., 2018).

Desinterés de las futuras generaciones, gran parte de los productores son gente de la tercera edad (> 60 años) y los jóvenes demuestran tener un desinterés debido a que han visto los problemas que han enfrentado sus padres, amigos, conocidos, etc., se percatan que el ingreso es poco redituable y requiere un gran esfuerzo físico (Higuera y Rivera, 2018).

Por esto, los productores proponen buscar fuentes económicas como fondo perdido, el cual consiste en apoyo económico del gobierno que se da a emprendedores o empresarios activos, este dinero no genera interés ni comisión, siempre y cuando se haya cumplido con el objetivo para el que fue usado. Existen varios programas que trabajan con fondos perdidos, por ejemplo: la Secretaría de Agricultura, Ganadería Desarrollo Rural, Pesca y Alimentación (SAGARPA), el Instituto Nacional de la Economía Social (INAES), la Secretaría del Desarrollo Social (SEDESOL), la Comisión Nacional para el desarrollo de los Pueblos Indígenas (CDI), el Instituto Nacional del Emprendedor (INADEM), la Comisión Nacional de Áreas Naturales Protegidas, entre otros. Los fondos perdidos pueden ser una buena alternativa para obtener el dinero que sea dirigido a la compra de la maquinaria (DOF, 2019). 
La utilización e implementación de programas de manejo integrado de plagas (MIP), propone una alternativa para reducir el daño económico causado por la presencia de plagas, procurando un equilibrio con el agroecosistema. Para ello se requiere personal capacitado que apoye a los caficultores del Estado, logrando un equilibrio entre rentabilidad y sostenibilidad. El programa proporciona elementos técnicos, metodológicos y pedagógicos que permiten la construcción de competencias necesarias para el desempeño eficaz en las actividades del control de plagas (Barrera et al., 2004).

También engloba temas como trabajos culturales, controles etológicos, biológicos y manuales, resistencia genética, cuidados del ambiente y aplicación de químicos. La capacitación tiene un costo económico que podría ser sufragado por aportaciones de las dependencias gubernamentales y la otra por caficultores. En este sentido, existe una brecha que se debe acotar, ya que según datos de Cuevas et al. (2012) la capacitación a productores está dada en $70.8 \%$ por técnicos, $17.7 \%$ por productores, $2.8 \%$ por instituciones académicas o de investigación y $1.5 \%$ otorgado por despachos; aunque la cobertura de asistencia técnica es muy baja, ya que 3\% del total nacional de unidades de producción disponen de este servicio.

Uno de los factores que puede intervenir para que los apoyos gubernamentales no lleguen a los productores es posiblemente a la falta de recursos para este sector o bien que el recurso no sea suficiente para cubrir a las necesidades del cultivo. Una alternativa seria presentar proyectos detallados para mejorar la cadena productiva, especialmente en los primeros eslabones (insumos, producción, acopio), es el trabajo colaborativo con enfoque a acciones participativas, tal como lo demostró Mills-Packo et al. (1991) en generar y transferir agrotecnología a los primeros eslabones de la cadena de café y otros cultivos en Hawái.

Por otra parte, las asesorías en toda la cadena productiva son fundamentales, especialmente en los primeros eslabones, debido a que los caficultores en la zona y en el país, tienen un nivel básico de educación, con una media de 5.6 años de educación (Rosales-Martínez et al., 2018). Para algunos, la educación es un impedimento para entender, aplicar o hasta generar nuevos modelos de negocio, estrategias de crecimiento, soluciones tecnológicas, etc., para otros pueden resultar retos que, combinando el conocimiento tradicional con tecnología, podrían agregar más valor a la producción que los haga más competitivos a nivel nacional e internacional (Benítez-García et al., 2015).

Por último, el modelo conceptual generado por los productores ante la falta de interés de las generaciones futuras para el cultivo del café (Figura 3). Parte de las soluciones planteadas por los actores son involucrar a niñas y niños en el proceso del café, apoyados desde la familia, la educación y programas por parte de las autoridades de los diferentes niveles, que incluya el otorgamiento de becas, talleres, actividades prácticas, entre otras, realizadas dentro y fuera de la escuela (Flores-López et al., 2018).

Estas actividades son consideradas como importantes por parte de los actores participantes, ya que la transmisión del conocimiento tradicional sobre el cuidado fitosanitario del cultivo, el proceso y momentos de siembra y cosecha, los procesos de transformación y hasta la parte de comercialización, hacia las nuevas generaciones, podría generar estructuras más consolidadas en la producción del aromático y fortalecer la cadena productiva (Higuera y Rivera, 2018). 


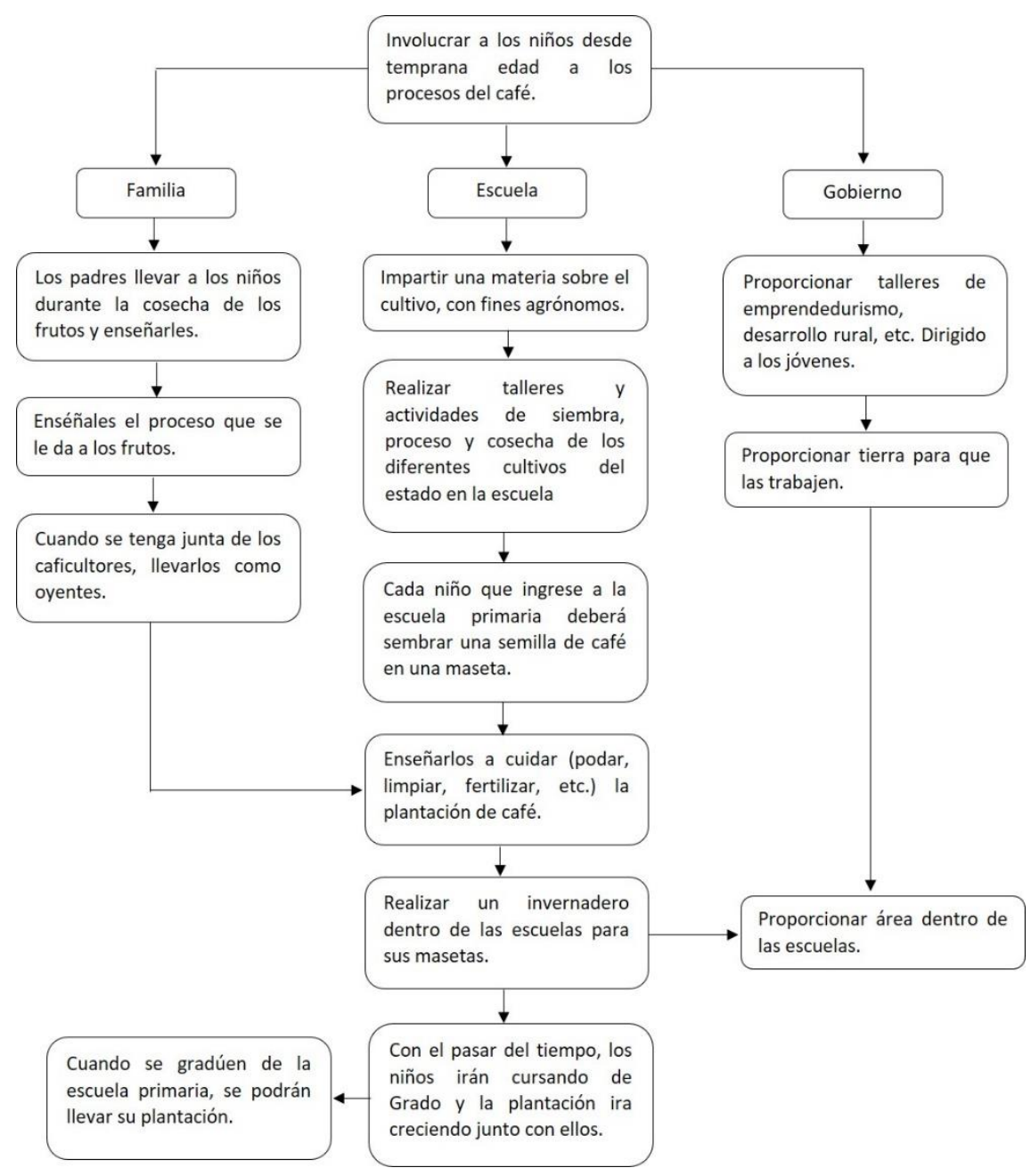

Figura 3. Modelo conceptual para interesar a las generaciones futuras.

Esta actividad de transmitir el conocimiento surge de la preocupación de que $70 \%$ de los productores de las principales regiones cafetaleras del país, podrían decidir cambiar de cultivos por otros más redituables (Rosales-Martínez et al., 2018).

\section{Conclusiones}

La metodología de sistemas blandos (SSM) puede brindar una visión general para comprender como se encuentra la situación de la cadena productiva del café en Guerrero. A través de SSM se dio un esbozo de los problemas que afectan al eslabón más débil de la cadena, que son los productores y la producción de su café, además de que se generaron algunas ideas para abordar la situación desde la base, así como crear soluciones que mejoren la situación de los productores y sus familias.

Existen varios interesados en aportar soluciones a los productores del estado; sin embargo, lleva tiempo el solo comprender los problemas que enfrentan los caficultores. Los resultados de la metodología proporcionan soluciones posibles como apoyo para generar un cambio radical a la situación que está impactando a la cadena productiva del café. 
En el proceso de evaluación se identificó que existen causas que se interrelacionan, por ejemplo, las plantaciones viejas son susceptibles a plagas y enfermedades, y no contar con apoyos gubernamentales como la capacitación de buenas prácticas sanitarias, pueden generar problemas de rendimientos y pérdidas económicas. Adicionalmente, si los caficultores del estado no cuentan con la tecnología necesaria para darle valor agregado al proceso y no obtener ganancias suficientes para vivir de sus cosechas, provoca que las generaciones futuras no tengan el interés de continuar cultivando la cereza del café y seguir con la tradición cafetalera.

Guerrero es un estado con superficie potencial para la producción de café, incluso más que otros estados como Oaxaca, Chiapas y Puebla; sin embargo, la pulverización de las tierras cafetaleras, las condiciones edafoecológicas y climáticas y la estructura sociopolítica, provocan que en el estado se tengan rendimientos por debajo de las $2 \mathrm{t} \mathrm{ha}^{-1}$. La metodología de sistemas blandos (SSM) puede impulsar diversas acciones que implican la participación entre actores de la cadena productiva del café, este reconocimiento en conjunto y la generación de alternativas pueden ser una base importante para la planeación de actividades que beneficien, no sólo la etapa de producción sino en toda la cadena productiva del aromático.

\section{Agradecimientos}

Este estudio forma parte del proyecto FORDECYT 'Estrategias multidisciplinarias para incrementar el valor agregado de las cadenas productivas del café, frijol, agave mezcalero, mango y productos acuícolas en la región Pacífico Sur a través de la ciencia, la tecnología y la innovación' con clave 292474 y fue desarrollado en el laboratorio de prospección tecnológica para el desarrollo innovador de los alimentos y la alimentación (PROTEAA) en el marco de los estudios de Maestría en Ciencias en Ingeniería de la Universidad Politécnica de Aguascalientes.

\section{Literatura citada}

Akaki, P. y Huacuja, F. 2006. Cadenas globales y café en México. Cuadernos geográficos de la Universidad de Granada. 38(1):69-86.

Barrera, J.; Parra, M.; Herrera, O.; Jarquín, R. y Pohlan, J. 2004. Plan estatal de manejo agroecológico del café en Chiapas: guía hacia una cafeticultura sustentable. Comisión para el desarrollo y fomento del café de Chiapas y el colegio de la frontera sur. Tapachula, Chiapas, México. 164 p.

Benítez-García, E.; Jaramillo-Villanueva, J.; Escobedo-Garrido, S. y Mora-Flores, S. 2015. Caracterización de la producción y del comercio de café en el municipio de Cuetzalan, Puebla. Agric. Soc. Des. 12(2):181-198.

Cezarino, L.; Liboni, L.; Oliveira, M. and Caldana, A. 2016. Soft systems methodology and interdisciplinarity in management education. Systems Res. Behavioral Sci. 33(2):278-288.

Checkland, P. 1999. Systems thinking. In: Rethinking management information systems. Currie, W. and Galliers, B. (Eds.). Oxford University Press, Oxford. 45-56 pp.

Checkland, P. and Poulter, J. 2006. Learning for action: a short definitive account of soft systems methodology and its use for practitioner, teachers, and students. John Wiley \& Sons Ltd, Chichester, West Sussex. 200 p. 
Contreras-Medina, D.; Sánchez, E.; Olvera, L. and Romero, Y. 2019. Technology roadmapping architecture based on knowledge management: case study for improved indigenous coffee production from Guerrero, Mexico. J. Sensors. 5860905.

Cruz, M. 2017. Productividad y rentabilidad potencial del café (Coffea arabica L.) en el trópico mexicano. Rev. Mex. Cienc. Agríc. 7(8):2011-2024.

Cuevas, V.; Baca-Moral, J.; Cervantes, F. y Aguilar, J. 2012. Asistencia técnica en el sector agropecuario en México: análisis del VIII censo agropecuario y forestal. Rev. Mex. Cienc. Agríc. 3(5):943-957.

Díaz, S. 2015. Cadenas productivas y redes de participación para el desarrollo: el café en México. Rev. Geog. Agríc. 55(2):57-73.

DOF. 2019. Diario oficial de la federación. Reglas de operación del fondo nacional de emprendedores para el ejercicio fiscal 2019. DOF: 28/02/2019. Gobierno de México.

Flores-López, M.; Sánchez-Osorio, E.; Pardo-Núñez, J. y Contreras-Medina, C. 2018. Documento estratégico características, tipología, estructura, trayectoria y competencias del entorno regional. Informe técnico preliminar del proyecto de problemas nacionales 2015. Centro de investigación y asistencia en tecnología y diseño del estado de Jalisco, AC. Consejo Nacional de Ciencia y Tecnología (CONACYT). 84 p.

Higuera, I. y Rivera, J. 2018. Chiapas: problemáticas del sector cafetalero. Centro de investigación y asistencia en tecnología y diseño del estado de Jalisco, AC. (Ed). 112 p.

Mills-Packo, A.; Wilson, K. and Rotar, P. 1991. Highlights from the use of the soft systems methodology to improve agrotechnology transfer in kona, Hawaii. Agric. Systems. 36(4):409-425.

Rosales-Martínez, V.; Martínez-Dávila, J.; Osorio-Acosta, F.; López-Romero, G.; Asiaín-Hoyos, A. y Estrella-Chulím, N. 2018. Aspectos culturales, sociales y productivos para una tipología de cafeticultores. Agric. Soc. Des. 15(1):47-61.

Santoyo, C.; Díaz, C. y Rodríguez P. 1995. Sistema agroindustrial café en México. Diagnóstico, problemática y alternativas. (Ed). Universidad Autónoma Chapingo (UACH). Texcoco, Estado de México. 157 p.

SIAP. 2019. Servicio de información agroalimentaria y pesquera. Cierre de la producción agrícolacafé cereza. https://nube.siap.gob.mx/

Wheeler, F.; Checkland, P. and Scholes, J. 2000. Soft systems methodology in action: including a 30-year retrospective. J. Operational Res. Soc. 51(5):648-649. 